\title{
A New Fifth-Order Finite Difference WENO Scheme for Dam-Break Simulations
}

\author{
Xiaogang $\mathrm{Li}^{1}$, Guodong $\mathrm{Li}^{1}{ }^{1, *}$ and Yongbin $\mathrm{Ge}^{2}$ \\ ${ }^{1}$ State Key Laboratory of Eco-Hydraulics in Northwest Arid Region, Xi'an \\ University of Technology, Xi'an, Shaanxi 710048, China \\ ${ }^{2}$ Institute of Applied Mathematics and Mechanics, Ningxia University, \\ Yinchuan, Ningxia 750021, China
}

Received 27 May 2019; Accepted (in revised version) 16 January 2020

\begin{abstract}
In this paper, a fifth-order weighted essentially nonoscillatory scheme is presented for simulating dam-break flows in a finite difference framework. The new scheme is a convex combination of two quadratic polynomials with a fourth-degree polynomial in a classical WENO fashion. The distinguishing feature of the present method is that the same five-point information is used but smaller absolute truncation errors and the same accuracy order in the smooth region are obtained. The new nonlinear weights are presented by Taylor expansion of the smoothness indicators of the small stencils to sustain the optimal fifth-order accuracy. The linear advection equation, nonlinear scalar Burgers equation, and one- and two-dimensional Euler equations are used to validate the high-order accuracy and excellent resolution of the presented method. Finally, one- and two-dimensional Saint-Venant equations are tested by using the new fifth-order scheme to simulate a dam-break flow.
\end{abstract}

AMS subject classifications: 65M60, 35L65

Key words: WENO scheme, smoothness indicators, shallow water equation, hyperbolic conservation laws.

\section{Introduction}

Dam-break simulations are very important in hydraulic engineering, as the work is related to people's lives and property safety. The governing equation of a dam break is the shallow water equation, also referred to as the Saint-Venant system [1]. It is widely applied in ocean and hydraulic engineering. This system describes the flow as a conservation law with an additional source. In one-dimensional space, the equation takes the

*Corresponding author.

Email: gdli2008@xaut.edu.cn (G. D. Li) 
form [2]

$$
\begin{aligned}
& \frac{\partial h}{\partial t}+\frac{\partial(h u)}{\partial x}=0, \\
& \frac{\partial(h u)}{\partial t}+\frac{\partial\left(h u^{2}+\frac{1}{2} g h^{2}\right)}{\partial x}=-g h \frac{\partial b}{\partial x},
\end{aligned}
$$

where $b$ is the vertical height of the bottom topography, $h$ denotes the water height, $u$ is the velocity of the fluid and $g$ is the gravitational constant. Because of the practical importance of the shallow water equation, studies on numerical methods for this system have attracted much attention in the past few years [1-4] and recently [5-9]. In the homogeneous case, the system is equivalent to a Euler system, which satisfies hyperbolic conservation laws.

One characteristic of the hyperbolic conservation laws is that they may develop discontinuities in the solution even if the initial conditions are smooth. Thus, classical numerical methods that depend on Taylor expansion fail to obtain an approximate solution for hyperbolic conservation laws. In the past few decades, many high-order finite difference or finite volume methods have been investigated to solve hyperbolic conservation laws [10-17]. However, high-order approximation leads to spurious oscillations in the solution. To overcome this phenomenon, total variation diminishing (TVD) schemes constructed by Harten $[10,18]$ are based on the principle that the total variation in the approximation of the numerical solution must be nonincreasing in time, however, it has been found that TVD schemes are at most first-order accurate near smooth extrema [19]. To improve the accuracy of TVD schemes, essentially non-oscillatory (ENO) and weighted essentially non-oscillatory (WENO) schemes have been applied quite successfully to solve problems with strong shocks, contact discontinuities and sophisticated smooth structures [20-30]. A series of ENO schemes were developed by Harten et al. [12] to solve the one-dimensional problem, where instead of using a single fixed stencil to approximate spatial fluxes, the ENO scheme used a set of candidate stencils determined by smoothness indicators. However, the ENO scheme is not effective, as such adaption of stencils is not necessary in smooth regions. Thus, the WENO scheme was introduced by Liu et al. [20] to overcome the drawbacks of the ENO scheme while maintaining robustness and high-order accuracy, in such schemes, spatial derivatives are calculated by using a convex combination of numerical fluxes associated with each candidate stencil. Jiang and Shu developed a classical method to calculate the smoothness indicators of the stencils, called the WENO-JS scheme, where the convergence accuracy of the WENO-JS scheme is fifth order in theory, but its actual rate of convergence is less than fifth order at critical points for many problems. The mapped WENO scheme (WENO-M) [31] was developed to have a formal fifth-order convergence at critical points of a smooth solution. This scheme uses a mapping function $k(\varepsilon)$, which renders the nonlinear weights closer to optimality to satisfy sufficient criteria for fifth-order convergence, however, the CPU cost is 1.25 times that of the WENO-JS scheme. By a simple combination of classical smoothness indicators, the WENO-Z scheme was presented in [15,32], which not only 
improves the convergence order at the critical points but also has the same CPU cost as the WENO-JS scheme, exhibiting a 0.25 reduction in CPU cost over that of the WENO-M scheme. The WENO-ZQ [33] scheme was developed by Zhu and Qiu for its simplicity and high-order accuracy, when a large, centered stencil permits optimal stability and accuracy to be reached, this method certainly reaches that stability and accuracy, especially when the solution on the stencil is rough.

In this paper, we aim to develop a new fifth-order finite difference WENO scheme. Like [33], we obtain a convex combination of a fourth-degree polynomial with two quadratic polynomials in the classical fashion. The new nonlinear weights are proposed by Taylor expansion for the smoothness indicators of the small stencils in the framework of a conventional WENO-Z scheme.

This paper is organized as follows. The new fifth-order finite difference WENO scheme is introduced in Section 2 in detail. In Section 3, some numerical tests are presented to verify the numerical accuracy and efficiency of the present WENO scheme, and the one- and two-dimensional dam-break problem is simulated. Concluding remarks are given in Section 4.

\section{A new high-order finite difference WENO scheme}

In this section, we present a new fifth-order finite difference weighted essentially nonoscillatory scheme. Without loss of generality, we consider a scalar hyperbolic conservation law equation in one dimension,

$$
\left\{\begin{array}{l}
u_{t}+f_{x}(u)=0, \\
u(x, 0)=u_{0}(x),
\end{array}\right.
$$

and the semidiscretized form of (2.1) is

$$
\frac{d u_{i}(t)}{d t}=L\left(u_{i}(t)\right)
$$

where $L(u)$ is the high-order spatial discrete formulation of $-f_{x}(u)$. Consider a uniform grid defined by the points $x_{i}=i \Delta x$, where the cells are $I_{i}=\left[x_{i-1 / 2}, x_{i+1 / 2}\right]$, cell size is denoted as $\Delta x=x_{i+1 / 2}-x_{i-1 / 2}$, and cell centers are denoted as $x_{i}=\frac{1}{2}\left(x_{i-1 / 2}+x_{i+1 / 2}\right)$, $i=1, \cdots, N$. The right-hand side of (2.2) can then be reformulated as

$$
L\left(u_{i}(t)\right)=-\frac{1}{\Delta x}\left(\hat{f}_{i+1 / 2}+\hat{f}_{i-1 / 2}\right),
$$

where $u_{i}(t)$ is defined as a nodal point value $u\left(x_{i}, t\right)$, and $\hat{f}_{i+1 / 2}$ is a numerical flux that has a fifth-order approximation of flux $f(u)$ at the cell boundary $x_{i+1 / 2}$ of cell $I_{i}$.

In general, the flux can be split into positive and negative parts by the flux vector splitting method:

$$
f(u)=f^{+}(u)+f^{-}(u),
$$




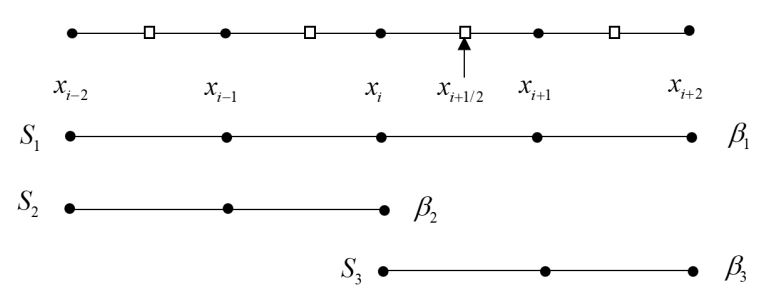

Figure 1: The computational uniform grid $x_{i}$ with the 5-point stencil $S_{1}$ and two 3-point stencils $S_{2}$ and $S_{3}$ used for the fifth-order WENO reconstruction step.

where $\frac{d f^{+}(u)}{d u} \geq 0, \frac{d f^{-}(u)}{d u} \leq 0$.

In this paper, we use Lax-Friedrichs flux vector splitting:

$$
f^{ \pm}(u)=\frac{1}{2}(f(u) \pm \alpha u)
$$

where $\alpha$ is taken as $\alpha=\max \left\{\left|f^{\prime}(u)\right|\right\}$ over the relevant $u$. $\hat{f}_{i+1 / 2}^{+}$and $\hat{f}_{i+1 / 2}^{-}$are the numerical fluxes at $x=x_{i+1 / 2}$ for positive and negative parts, respectively. Thus,

$$
\hat{f}_{i+1 / 2}=\hat{f}_{i+1 / 2}^{+}+\hat{f}_{i+1 / 2}^{-} \text {. }
$$

Hereafter, we only describe how $\hat{f}_{i+1 / 2}^{+}$is approximated because $\hat{f}_{i+1 / 2}^{-}$is symmetric to $\hat{f}_{i+1 / 2}^{+}$with respect to $x=x_{i+1 / 2}$. We will drop the " + "sign in the superscript for simplicity.

The large stencil (Fig. 1) is chosen as $S_{1}=\left\{x_{i-2}, x_{i-1}, x_{i}, x_{i+1}, x_{i+2}\right\}$. It is easy to obtain a fourth-degree reconstructed polynomial $q_{1}(x)$ :

$$
\begin{aligned}
q_{1}(x)=f_{i} & +\frac{f_{i+2}-8 f_{i+1}+8 f_{i-1}-f_{i-2}}{12 \Delta x}\left(x-x_{i}\right) \\
& -\frac{f_{i+2}-16 f_{i+1}+30 f_{i}-16 f_{i-1}+f_{i-2}}{24 \Delta x^{2}}\left(x-x_{i}\right)^{2} \\
& +\frac{f_{i+2}-2 f_{i+1}+2 f_{i-1}-f_{i-2}}{12 \Delta x^{3}}\left(x-x_{i}\right)^{3} \\
& +\frac{f_{i+2}-4 f_{i+1}+6 f_{i}-4 f_{i-1}+f_{i-2}}{24 \Delta x^{4}}\left(x-x_{i}\right)^{4} \\
& -\frac{1}{24} \Delta x^{2} \frac{\partial^{2} p_{1}(x)}{\partial x^{2}}+\frac{1}{5760} \Delta x^{4} \frac{\partial^{4} p_{1}(x)}{\partial x^{4}},
\end{aligned}
$$

where $p_{1}(x)$ is a fourth-degree Lagrangian interpolation polynomial. This reconstructed polynomial is also given in [33] with a different expression, where the fifth-order accurate numerical flux is given by

$$
\hat{f}_{i+1 / 2}^{1}=q_{1}\left(x_{i+1 / 2}\right)=\frac{1}{30} f_{i-2}-\frac{13}{60} f_{i-1}+\frac{47}{60} f_{i}+\frac{9}{20} f_{i+1}-\frac{1}{20} f_{i+2} .
$$


Choose two small stencils (Fig. 1): $S_{2}=\left\{x_{i-2}, x_{i-1}, x_{i}\right\}$ and $S_{3}=\left\{x_{i}, x_{i+1}, x_{i+2}\right\}$. It is also easy to obtain the two quadratic polynomials:

$$
\begin{aligned}
q_{2}(x)= & f_{i-1}+\frac{f_{i}-f_{i-2}}{2 \Delta x}\left(x-x_{i-1}\right)-\frac{f_{i}-2 f_{i-1}+f_{i-2}}{2 \Delta x^{2}}\left(x-x_{i-1}\right)^{2} \\
& -\frac{1}{24} \Delta x^{2} \frac{\partial^{2} p_{2}(x)}{\partial x^{2}}, \\
q_{3}(x)= & f_{i+1}+\frac{f_{i+2}-f_{i}}{2 \Delta x}\left(x-x_{i+1}\right)-\frac{f_{i+2}-2 f_{i+1}+f_{i}}{2 \Delta x^{2}}\left(x-x_{i+1}\right)^{2} \\
& -\frac{1}{24} \Delta x^{2} \frac{\partial^{2} p_{3}(x)}{\partial x^{2}}
\end{aligned}
$$

where $p_{2}(x)$ and $p_{3}(x)$ are quadratic Lagrangian interpolation polynomials. The thirdorder accurate numerical fluxes are given by

$$
\begin{aligned}
& \hat{f}_{i+1 / 2}^{2}=q_{2}\left(x_{i+1 / 2}\right)=\frac{1}{3} f_{i-2}-\frac{7}{6} f_{i-1}+\frac{11}{6} f_{i}, \\
& \hat{f}_{i+1 / 2}^{3}=q_{3}\left(x_{i+1 / 2}\right)=\frac{1}{3} f_{i}+\frac{5}{6} f_{i+1}-\frac{1}{6} f_{i+2} .
\end{aligned}
$$

The smoothness indicators are $\beta_{n}(n=1,2,3)$, which are used to measure the smoothness of the functions $p_{n}(x)$. We use the smoothness indicators in [21]:

$$
\beta_{n}=\sum_{\alpha=1}^{r} \int_{I_{i}} \Delta x^{2 \alpha-1}\left(\frac{d^{\alpha} p_{n}(x)}{d x^{\alpha}}\right)^{2} d x, \quad n=1,2,3 .
$$

The associated explicit expressions of smoothness indicators are

$$
\begin{aligned}
& \beta_{1}=\frac{1}{144}\left(f_{i-2}-8 f_{i-1}+8 f_{i+1}-f_{i+2}\right)^{2} \\
& +\frac{1}{15600}\left(-11 f_{i-2}+174 f_{i-1}-362 f_{i}+174 f_{i+1}-11 f_{i+2}\right)^{2} \\
& +\frac{781}{2880}\left(-f_{i-2}+2 f_{i-1}-2 f_{i+1}+f_{i+2}\right)^{2} \\
& +\frac{1421461}{1310400}\left(f_{i-2}-4 f_{i-1}+6 f_{i}-4 f_{i+1}+f_{i+2}\right)^{2}, \\
& \beta_{2}=\frac{13}{12}\left(f_{i-2}-2 f_{i-1}+f_{i}\right)^{2}+\frac{1}{4}\left(f_{i-2}-4 f_{i-1}+3 f_{i}\right)^{2} \text {, } \\
& \beta_{3}=\frac{13}{12}\left(f_{i}-2 f_{i+1}+f_{i+2}\right)^{2}+\frac{1}{4}\left(3 f_{i}-4 f_{i+1}+f_{i+2}\right)^{2} .
\end{aligned}
$$

The Taylor series expansions of the smoothness indicators about $f_{i}$ are obtained:

$$
\begin{aligned}
\beta_{1} & =\Delta x^{2}\left(f_{i}^{\prime}\right)^{2}+\frac{13}{12} \Delta x^{4}\left(f_{i}^{\prime \prime}\right)^{2}+\frac{1}{5040} \Delta x^{6}\left[5467\left(f_{i}^{\prime \prime}\right)^{2}-14 f_{i}^{\prime \prime} f_{i}^{(4)}-336 f_{i}^{\prime} f_{i}^{(5)}\right]+\mathcal{O}\left(\Delta x^{8}\right) \\
& =\Delta x^{2}\left(f_{i}^{\prime}\right)^{2}\left[1+\mathcal{O}\left(\Delta x^{2}\right)\right]=\mathcal{O}\left(\Delta x^{2}\right),
\end{aligned}
$$




$$
\begin{aligned}
\beta_{2} & =\Delta x^{2}\left(f_{i}^{\prime}\right)^{2}+\Delta x^{4}\left[\frac{13}{12}\left(f_{i}^{\prime \prime}\right)^{2}-\frac{2}{3} f_{i}^{\prime} f_{i}^{\prime \prime \prime}\right]+\Delta x^{5}\left(-\frac{13}{6} f_{i}^{\prime \prime} f_{i}^{\prime \prime \prime}+\frac{1}{2} f_{i}^{\prime} f_{i}^{(4)}\right)+\mathcal{O}\left(\Delta x^{6}\right) \\
& =\Delta x^{2}\left(f_{i}^{\prime}\right)^{2}\left[1+\mathcal{O}\left(\Delta x^{2}\right)\right]=\mathcal{O}\left(\Delta x^{2}\right) \\
\beta_{3} & =\Delta x^{2}\left(f_{i}^{\prime}\right)^{2}+\Delta x^{4}\left[\frac{13}{12}\left(f_{i}^{\prime \prime}\right)^{2}-\frac{2}{3} f_{i}^{\prime} f_{i}^{\prime \prime \prime}\right]+\Delta x^{5}\left(\frac{13}{6} f_{i}^{\prime \prime} f_{i}^{\prime \prime \prime}-\frac{1}{2} f_{i}^{\prime} f_{i}^{(4)}\right)+\mathcal{O}\left(\Delta x^{6}\right) \\
& =\Delta x^{2}\left(f_{i}^{\prime}\right)^{2}\left[1+\mathcal{O}\left(\Delta x^{2}\right)\right]=\mathcal{O}\left(\Delta x^{2}\right) .
\end{aligned}
$$

From Eqs. (2.13a)-(2.13c), we can obtain

$$
\begin{aligned}
& \beta_{1}-\beta_{2}=\frac{2}{3} \Delta x^{4} f_{i}^{\prime} f_{i}^{\prime \prime \prime}+\Delta x^{5}\left(\frac{13}{6} f_{i}^{\prime \prime} f_{i}^{\prime \prime \prime}-\frac{1}{2} f_{i}^{\prime} f_{i}^{(4)}\right)+\mathcal{O}\left(\Delta x^{6}\right) \\
& =\left\{\begin{array}{lll}
\mathcal{O}\left(\Delta x^{4}\right), & f_{i}^{\prime} \neq 0, & f_{i}^{\prime \prime} \neq 0, \\
\mathcal{O}\left(\Delta x^{5}\right), & f_{i}^{\prime}=0, & f_{i}^{\prime \prime} \neq 0,
\end{array}\right. \\
& \beta_{1}-\beta_{3}=\frac{2}{3} \Delta x^{4} f_{i}^{\prime} f_{i}^{\prime \prime \prime}+\Delta x^{5}\left(-\frac{13}{6} f_{i}^{\prime \prime} f_{i}^{\prime \prime \prime}+\frac{1}{2} f_{i}^{\prime} f_{i}^{(4)}\right)+\mathcal{O}\left(\Delta x^{6}\right) \\
& =\left\{\begin{array}{lll}
\mathcal{O}\left(\Delta x^{4}\right), & f_{i}^{\prime} \neq 0, & f_{i}^{\prime \prime} \neq 0, \\
\mathcal{O}\left(\Delta x^{5}\right), & f_{i}^{\prime}=0, & f_{i}^{\prime \prime} \neq 0 .
\end{array}\right.
\end{aligned}
$$

It is easy to verify

$$
\begin{aligned}
\tau & =\left(\frac{\left|\beta_{1}-\beta_{2}\right|+\left|\beta_{1}-\beta_{3}\right|}{2}\right)^{2} \\
& =\left\{\begin{array}{lll}
\mathcal{O}\left(\Delta x^{8}\right), & f_{i}^{\prime} \neq 0, & f_{i}^{\prime \prime} \neq 0, \\
\mathcal{O}\left(\Delta x^{10}\right), & f_{i}^{\prime}=0, & f_{i}^{\prime \prime} \neq 0 .
\end{array}\right.
\end{aligned}
$$

We define

$$
\omega_{n}=\frac{\bar{\omega}_{n}}{\sum_{l=1}^{3} \bar{\omega}_{l}}, \quad \bar{\omega}_{n}=\gamma_{n}\left(1+\frac{\tau}{\varepsilon+\beta_{n}}\right), \quad n=1,2,3 .
$$

Here, $\varepsilon$ is a small positive number so that the denominator does not become zero. In the smooth region, the following equation is satisfied

$$
\frac{\tau}{\varepsilon+\beta_{n}}=\mathcal{O}\left(\Delta x^{6}\right), \quad n=1,2,3 .
$$

Therefore, $\omega_{n}(n=1,2,3)$ satisfy the accuracy order condition. We take $\varepsilon=10^{-6}$ in our computation.

The new reconstruction of the numerical flux at $x_{i+1 / 2}$ is given by

$$
\begin{aligned}
\hat{f}_{i+1 / 2}=\omega_{1} & \left(\frac{1}{\gamma_{1}} q_{1}\left(x_{i+1 / 2}\right)-\frac{\gamma_{2}}{\gamma_{1}} q_{2}\left(x_{i+1 / 2}\right)-\frac{\gamma_{3}}{\gamma_{1}} q_{3}\left(x_{i+1 / 2}\right)\right) \\
& +\omega_{2} q_{2}\left(x_{i+1 / 2}\right)+\omega_{3} q_{3}\left(x_{i+1 / 2}\right) .
\end{aligned}
$$

The right side of (2.18) is different from the classical WENO scheme, with Eq. (2.18) clearly holding for arbitrarily chosen linear weights $\gamma_{1}, \gamma_{2}$ and $\gamma_{3}$, satisfying $\gamma_{1}+\gamma_{2}+\gamma_{3}=$ 
1. The purpose of this approach is to offer high-order approximation at point $x_{i+1 / 2}$ to numerical flux in the smooth region, if not, the scheme would degrade its optimal fifthorder accuracy. In this way, the scheme can sustain high-order accuracy in the smooth region and keep the shock transition sharp in the non-smooth region.

In the semidiscretized scheme of (2.2), time marching is used for the third-order total variation diminishing Runge-Kutta scheme (RK-TVD):

$$
\left\{\begin{array}{l}
u^{(1)}=u^{n}+\Delta t L\left(u^{n}\right), \\
u^{(2)}=\frac{1}{4}\left(3 u^{n}+u^{(1)}+\Delta t L\left(u^{(1)}\right)\right), \\
u^{(3)}=\frac{1}{3}\left(u^{n}+2 u^{(2)}+2 \Delta t L\left(u^{(2)}\right)\right) .
\end{array}\right.
$$

\section{Numerical experiments}

In this section, we compare the numerical performance of the new WENO scheme, termed WENO-Present, with the classical WENO-JS and the WENO-ZQ. First, a linear advection equation with smooth exact solutions is used to test the order of the accuracy of the WENO-Present scheme. Second, the good resolution of the WENO-Present scheme is tested by a large gradient problem of the linear advection equation. Third, we solve the nonlinear scalar Burgers equation by the WENO-Present scheme. Fourth, one- and two-dimensional systems of the Euler equation are tested by the new scheme. Finally, the dam-break model of one- and two-dimensional problems are simulated by the WENOPresent scheme. To test whether the choice of $\gamma$ would pollute the optimal order accuracy of the WENO-Present scheme, we first set the following types of linear weights in Section 3.1: (1) $\gamma_{1}=0.98, \gamma_{2}=0.01$, and $\gamma_{3}=0.01$; (2) $\gamma_{1}=1.0 / 3.0, \gamma_{2}=1.0 / 3.0$, and $\gamma_{3}=1.0 / 3.0$; (3) $\gamma_{1}=0.01, \gamma_{2}=0.495$, and $\gamma_{3}=0.495$

\subsection{Linear advection example with smooth exact solution and periodic boundary conditions}

For

$$
\frac{\partial u}{\partial t}+\frac{\partial u}{\partial x}=0, \quad x \in[0,1]
$$

with the initial condition

$$
u(x, 0)=\sin (2 \pi x)
$$

and the exact solution

$$
u(x, t)=\sin (2 \pi(x-t)) .
$$

Tables 1 and 2 show the errors and convergence orders of the WENO-Present scheme with different types of linear weights for Example 3.1 at $t=1$. The WENO-Present scheme with different types of linear weights achieves the fifth order of accuracy, and the truncation error of WENO-Present(1) is smaller than that of the others. 
Table 1: $L_{2}$ error and convergence order of the WENO-Present scheme with different types of linear weights for Example 3.1.

\begin{tabular}{||c|cccccc||}
\hline \multirow{2}{*}{ Grid Points } & \multicolumn{2}{|c|}{ WENO-Present(1) } & \multicolumn{2}{c|}{ WENO-Present(2) } & \multicolumn{2}{c||}{ WENO-Present(3) } \\
\cline { 2 - 7 } & Error & Order & Error & Order & Error & Order \\
\hline 10 & $9.60 \mathrm{e}-3$ & & $8.48 \mathrm{e}-3$ & & $1.02 \mathrm{e}-2$ & \\
20 & $2.24 \mathrm{e}-4$ & 5.42 & $2.33 \mathrm{e}-4$ & 5.19 & $2.24 \mathrm{e}-4$ & 5.40 \\
40 & $7.07 \mathrm{e}-6$ & 5.00 & $7.11 \mathrm{e}-6$ & 5.03 & $7.16 \mathrm{e}-6$ & 5.08 \\
80 & $2.21 \mathrm{e}-7$ & 5.00 & $2.21 \mathrm{e}-7$ & 5.01 & $2.21 \mathrm{e}-7$ & 5.01 \\
160 & $6.92 \mathrm{e}-9$ & 5.00 & $6.92 \mathrm{e}-9$ & 5.00 & $6.92 \mathrm{e}-9$ & 5.00 \\
\hline
\end{tabular}

Table 2: $L_{\infty}$ error and convergence order of the WENO-Present scheme with different types of linear weights for Example 3.1.

\begin{tabular}{||c|cccccc||}
\hline \multirow{2}{*}{ Grid Points } & \multicolumn{2}{|c|}{ WENO-Present(1) } & \multicolumn{2}{c|}{ WENO-Present(2) } & \multicolumn{2}{c||}{ WENO-Present(3) } \\
\cline { 2 - 7 } & Error & Order & Error & Order & Error & Order \\
\hline 10 & $6.86 \mathrm{e}-3$ & & $1.23 \mathrm{e}-2$ & & $1.47 \mathrm{e}-2$ & \\
20 & $3.13 \mathrm{e}-4$ & 4.45 & $3.23 \mathrm{e}-4$ & 5.25 & $3.45 \mathrm{e}-4$ & 5.41 \\
40 & $9.97 \mathrm{e}-6$ & 4.97 & $1.02 \mathrm{e}-5$ & 4.98 & $1.05 \mathrm{e}-5$ & 5.04 \\
80 & $3.12 \mathrm{e}-7$ & 5.00 & $3.15 \mathrm{e}-7$ & 5.02 & $3.17 \mathrm{e}-7$ & 5.05 \\
160 & $9.78 \mathrm{e}-9$ & 5.00 & $9.80 \mathrm{e}-9$ & 5.00 & $9.82 \mathrm{e}-9$ & 5.01 \\
\hline
\end{tabular}

Fig. 2 shows that the WENO-Present(1) scheme needs less CPU time than WENOPresent(2) and WENO-Present(3) to obtain the same $L_{2}$ and $L_{\infty}$ errors. Therefore, the WENO-Present(1) scheme is the most efficient, and it is adopted in later sections.

Tables 3 and 4 show the errors and convergence orders for Example 3.1 at $t=1$. We can see that the WENO-Present scheme achieves its designed order of accuracy. Additionally, the $L_{2}$ and $L_{\infty}$ errors of the WENO-Present scheme are less than those of the WENO-JS and WENO-ZQ schemes.

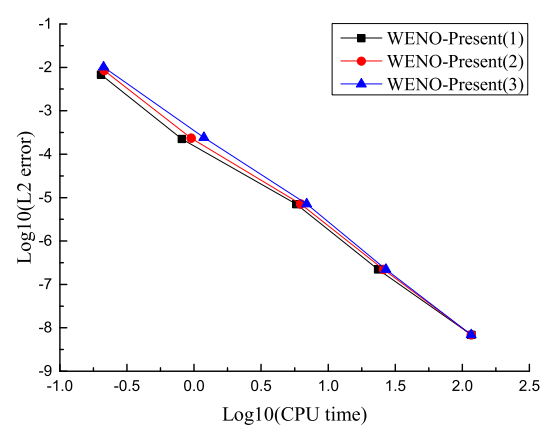

(a) $L_{2}$ error

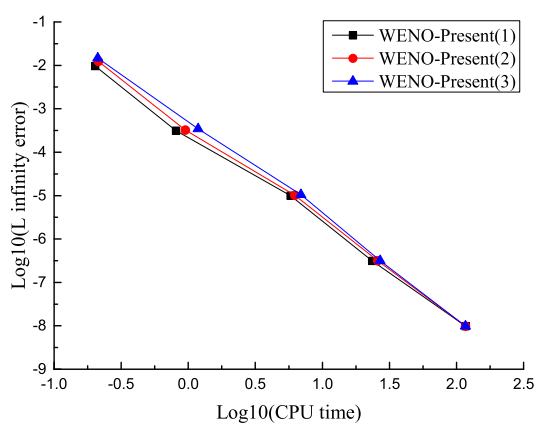

(b) $L_{\infty}$ error

Figure 2: Computing time and error of the WENO-Present scheme with different linear weights for Example 3.1. 
Table 3: A comparison study of $L_{2}$ error and convergence order of Example 3.1 at $t=1$.

\begin{tabular}{||c|cccccc||}
\hline \multirow{2}{*}{ Grid Points } & \multicolumn{2}{|c}{ WENO-JS } & \multicolumn{2}{c|}{ WENO-ZQ } & \multicolumn{2}{c||}{ WENO-Present } \\
\cline { 2 - 7 } & Error & Order & Error & Order & Error & Order \\
\hline 10 & $3.81 \mathrm{e}-2$ & & $4.76 \mathrm{e}-2$ & & $9.60 \mathrm{e}-3$ & \\
20 & $1.92 \mathrm{e}-3$ & 4.31 & $1.46 \mathrm{e}-3$ & 5.00 & $2.24 \mathrm{e}-4$ & 5.42 \\
40 & $5.83 \mathrm{e}-5$ & 5.04 & $4.52 \mathrm{e}-5$ & 5.01 & $7.07 \mathrm{e}-6$ & 5.00 \\
80 & $1.76 \mathrm{e}-6$ & 5.05 & $1.38 \mathrm{e}-6$ & 5.03 & $2.21 \mathrm{e}-7$ & 5.00 \\
160 & $5.41 \mathrm{e}-8$ & 5.02 & $4.32 \mathrm{e}-8$ & 5.00 & $6.92 \mathrm{e}-9$ & 5.00 \\
\hline
\end{tabular}

Table 4: A comparison study of $L_{\infty}$ error and convergence order of Example 3.1 at $t=1$.

\begin{tabular}{||c|cccccc||}
\hline \multirow{2}{*}{ Grid Points } & \multicolumn{2}{|c}{ WENO-JS } & \multicolumn{2}{c|}{ WENO-ZQ } & \multicolumn{2}{c||}{ WENO-Present } \\
\cline { 2 - 7 } & Error & Order & Error & Order & Error & Order \\
\hline 10 & $5.08 \mathrm{e}-2$ & & $6.02 \mathrm{e}-2$ & & $6.86 \mathrm{e}-3$ & \\
20 & $2.79 \mathrm{e}-3$ & 4.19 & $2.23 \mathrm{e}-3$ & 4.75 & $3.13 \mathrm{e}-4$ & 4.45 \\
40 & $9.82 \mathrm{e}-5$ & 4.83 & $6.85 \mathrm{e}-5$ & 5.02 & $9.97 \mathrm{e}-6$ & 4.97 \\
80 & $3.04 \mathrm{e}-6$ & 5.01 & $2.11 \mathrm{e}-6$ & 5.02 & $3.12 \mathrm{e}-7$ & 5.00 \\
160 & $9.45 \mathrm{e}-8$ & 5.01 & $6.57 \mathrm{e}-8$ & 5.00 & $9.78 \mathrm{e}-9$ & 5.00 \\
\hline
\end{tabular}

\subsection{Linear advection example for the large gradient problem and periodic boundary conditions}

For

$$
\frac{\partial u}{\partial t}+\frac{\partial u}{\partial x}=0, \quad x \in[-1,1]
$$

with the initial condition

$$
u(x, 0)=e^{-300 x^{2}}
$$

and the exact solution

$$
u(x, t)=e^{-300(x-t)^{2}} .
$$

Fig. 3 shows that the dissipation of the WENO-Present scheme is less than that of the WENO-JS and WENO-ZQ schemes. We can also see that the behavior of the WENOPresent scheme is better than that of the others. Thus, the WENO-Present scheme is better for simulating large gradient problems. Fig. 4 shows that the absolute error of the WENO-Present scheme is minimal, therefore, the WENO-Present scheme has a high resolution.

\subsection{Nonlinear scalar Burgers equation with periodic boundary conditions}

For

$$
\frac{\partial u}{\partial t}+\frac{\partial\left(\frac{u^{2}}{2}\right)}{\partial x}=0, \quad x \in[-1,1]
$$




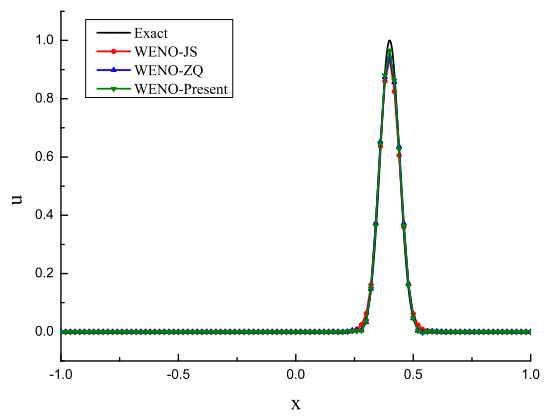

(a) Solution

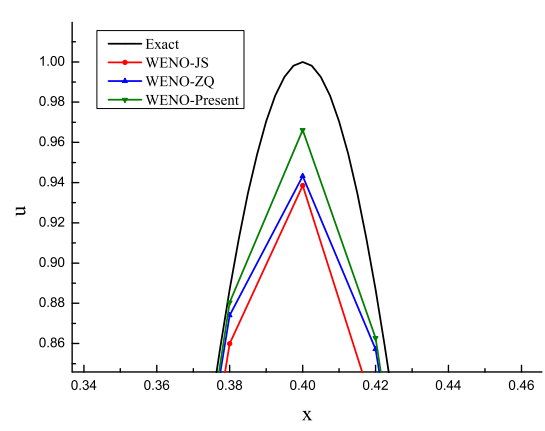

(b) Enlarged portion

Figure 3: Numerical solution of Example 3.2 computed by the WENO-JS, WENO-ZQ and WENO-Present schemes. The figures show the solution at $t=0.5$. $N=100$ points were used.

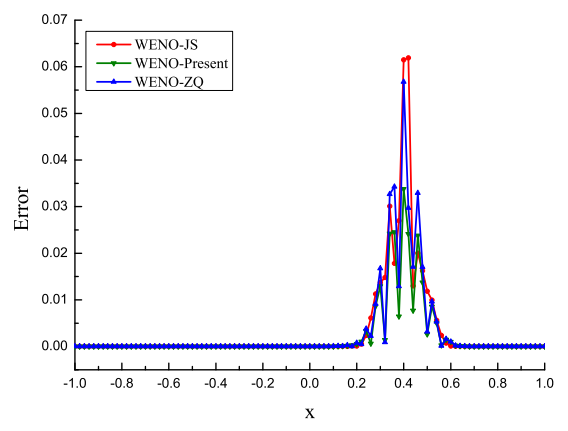

(a) Error

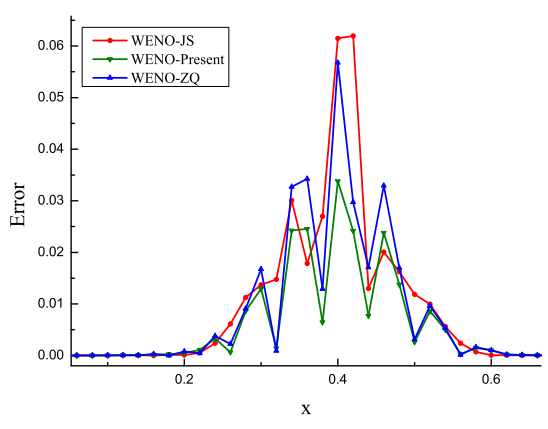

(b) Enlarged portion

Figure 4: Absolute error of Example 3.2 computed by the WENO-JS, WENO-ZQ and WENO-Present schemes. The figures show the solution at $t=0.5$. $N=100$ points were used.

with the initial condition

$$
u(x, 0)=\frac{1}{2}+\sin (\pi x) .
$$

The exact solution is smooth up to $t=1 / \pi$, then it develops a moving shock that interacts with a rarefaction wave. When $t=0.15$, the solution is still smooth. The errors and numerical orders of accuracy by the WENO-JS, WENO-ZQ and WENO-Present schemes are shown in Tables 5 and 6 . We can see that all the schemes achieve their designed order of accuracy. The WENO-Present and WENO-ZQ schemes produce fewer truncation errors than the WENO-JS scheme, and the error of WENO-Present is nearly the same as that of WENO-ZQ for this smooth solution.

When $t=0.5$, the solution is discontinuous, the approximate solutions are then computed by the WENO-JS, WENO-ZQ and WENO-Present schemes for the initial condi- 
Table 5: A comparison study of $L_{2}$ error and convergence order of Example 3.3 at $t=0.15$.

\begin{tabular}{||c|cccccc||}
\hline \multirow{2}{*}{ Grid Points } & \multicolumn{2}{|c}{ WENO-JS } & \multicolumn{2}{c|}{ WENO-ZQ } & \multicolumn{2}{c||}{ WENO-Present } \\
\cline { 2 - 7 } & Error & Order & Error & Order & Error & Order \\
\hline 10 & $2.96 \mathrm{e}-2$ & & $2.92 \mathrm{e}-2$ & & $2.69 \mathrm{e}-2$ & \\
20 & $3.42 \mathrm{e}-3$ & 3.11 & $2.67 \mathrm{e}-3$ & 3.45 & $2.64 \mathrm{e}-3$ & 3.35 \\
40 & $1.78 \mathrm{e}-4$ & 4.26 & $1.49 \mathrm{e}-4$ & 4.16 & $1.50 \mathrm{e}-4$ & 4.12 \\
80 & $6.36 \mathrm{e}-6$ & 4.81 & $5.45 \mathrm{e}-6$ & 4.77 & $5.44 \mathrm{e}-6$ & 4.79 \\
160 & $2.87 \mathrm{e}-7$ & 4.45 & $1.99 \mathrm{e}-7$ & 4.76 & $1.98 \mathrm{e}-7$ & 4.78 \\
320 & $9.90 \mathrm{e}-9$ & 4.86 & $5.68 \mathrm{e}-9$ & 5.12 & $5.68 \mathrm{e}-9$ & 5.12 \\
\hline
\end{tabular}

Table 6: A comparison study of $L_{\infty}$ error and convergence order of Example 3.3 at $t=0.15$.

\begin{tabular}{||c|cccccc||}
\hline \multirow{2}{*}{ Grid Points } & \multicolumn{2}{|c}{ WENO-JS } & \multicolumn{2}{c|}{ WENO-ZQ } & \multicolumn{2}{c||}{ WENO-Present } \\
\cline { 2 - 7 } & Error & Order & Error & Order & Error & Order \\
\hline 10 & $4.45 \mathrm{e}-2$ & & $4.06 \mathrm{e}-2$ & & $3.85 \mathrm{e}-2$ & \\
20 & $5.65 \mathrm{e}-3$ & 2.98 & $4.25 \mathrm{e}-3$ & 3.26 & $4.26 \mathrm{e}-3$ & 3.18 \\
40 & $4.03 \mathrm{e}-4$ & 3.81 & $3.47 \mathrm{e}-4$ & 3.61 & $3.47 \mathrm{e}-4$ & 3.62 \\
80 & $1.58 \mathrm{e}-5$ & 4.67 & $1.32 \mathrm{e}-5$ & 4.72 & $1.32 \mathrm{e}-5$ & 4.72 \\
160 & $1.20 \mathrm{e}-6$ & 3.72 & $7.48 \mathrm{e}-7$ & 4.14 & $7.47 \mathrm{e}-7$ & 4.14 \\
320 & $6.28 \mathrm{e}-8$ & 4.26 & $2.57 \mathrm{e}-8$ & 4.86 & $2.57 \mathrm{e}-8$ & 4.86 \\
\hline
\end{tabular}

tions, Figs. 5 and 6 show the results with grid points $N=40$ and $N=80$ respectively. The exact solution is calculated by the WENO-JS scheme with 2000 grid points. The shocks are very well captured by all the schemes, but the WENO-Present scheme performs the best.

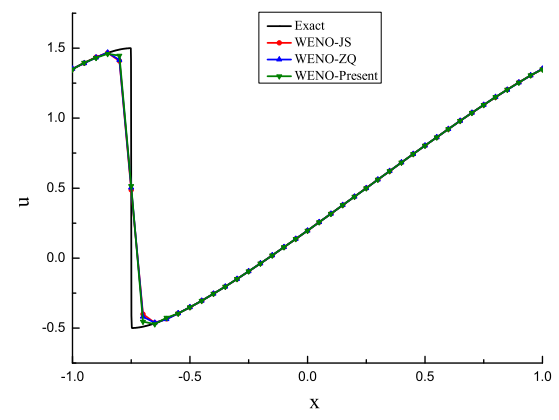

(a) Solution

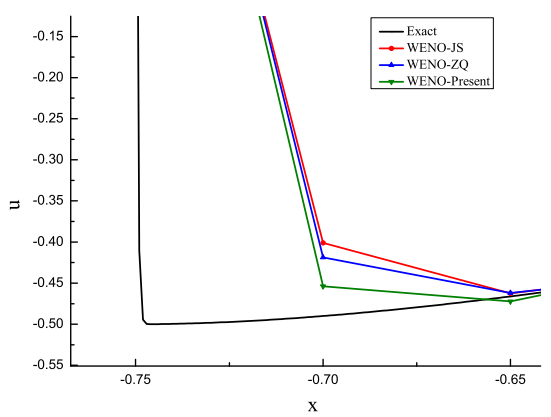

(b) Enlarged portion

Figure 5: Numerical solution of Example 3.3 computed by the WENO-JS, WENO-ZQ and WENO-Present schemes. The figures show the solution at $t=0.5$. $N=40$ points were used. 


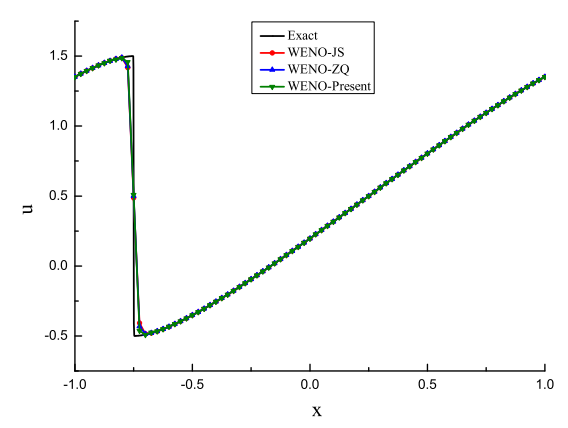

(a) Solution

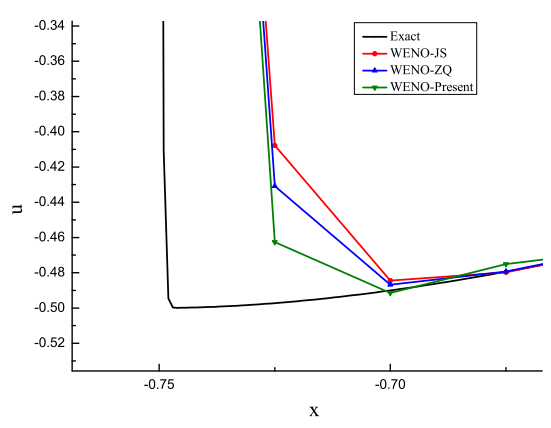

(b) Enlarged portion

Figure 6: Numerical solution of Example 3.3 computed by the WENO-JS, WENO-ZQ and WENO-Present schemes. The figures show the solution at $t=0.5$. $N=80$ points were used.

\subsection{One-dimensional Euler equations}

In this section, we consider 1D Euler equations since one of the main application areas of WENO schemes is compressible gas dynamics:

$$
\left(\begin{array}{c}
\rho \\
\rho u \\
E
\end{array}\right)_{t}+\left(\begin{array}{c}
\rho u \\
\rho u^{2}+p \\
(E+p) u
\end{array}\right)_{x}=0
$$

where $\rho, u, p$, and $E$ are density, velocity, pressure, and total energy, respectively. The system of equations is closed by the equation of states for an ideal polytropic gas: $E=$ $\frac{p}{\gamma-1}+\frac{1}{2} \rho u^{2}$, where the ratio of specific heats is $\gamma=1.4$.

The following two initial conditions combined with Euler equations (3.9) are considered.

1. Lax problem:

$$
(\rho, u, p)= \begin{cases}(0.445,0.698,3.528), & -0.5 \leq x \leq 0, \\ (0.5,0,0.571), & 0<x \leq 0.5\end{cases}
$$

The final computing time is $t=0.16$. We compare the performances of the exact solution and the computed density obtained with the WENO-JS, WENO-ZQ and WENO-Present schemes by using 200 grid points, the exact solution is the numerical solution of the WENO-JS scheme with 2000 grid points. The results for different schemes are shown in Fig. 7, we observe that the results obtained by WENO-ZQ and WENO-Present schemes work a little overshoot near the shock for this problem.

2. Shu-Osher problem: For

$$
(\rho, u, p)= \begin{cases}(3.857143,2.629369,10.33333), & -5 \leq x \leq-4 \\ (1+0.2 \sin (5 x-5), 0,1), & -4<x \leq 5\end{cases}
$$




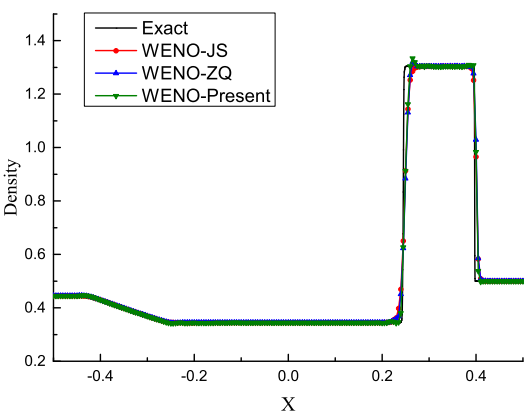

(a) Density

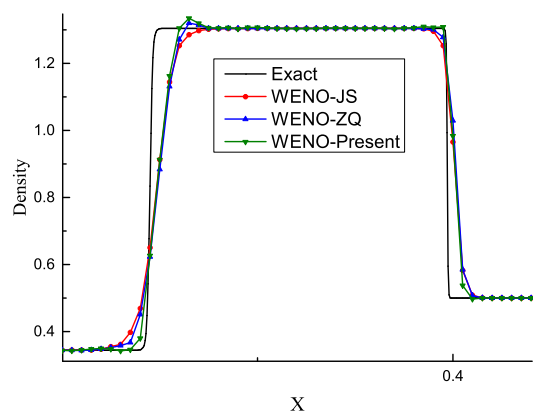

(b) Enlarged portion

Figure 7: Numerical solution of the Riemann problem of Lax computed by the WENO-JS, WENO-ZQ and WENO-Present schemes. The figures show the density of the solution at $t=0.16 . N=200$ points were used.

The Shu-Osher problem describes shock interaction with entropy waves. It is a typical problem with a moving shock interacting with sine waves in density, which means that the solution contains both shocks and complex smooth region structures. The final time is $t=1.8$. In Fig. 8 , we present the computed density against the exact solution, where the exact solution is computed by the WENO-JS scheme with 2000 grid points. We can see that the WENO-Present scheme shows a higher resolution than the WENO-JS and WENO-ZQ schemes.

\subsection{Two-dimensional Euler equations}

In this example, we consider the 2D Euler equation:

$$
\left(\begin{array}{c}
\rho \\
\rho u \\
\rho v \\
E
\end{array}\right)_{t}+\left(\begin{array}{c}
\rho u \\
\rho u^{2}+p \\
\rho u v \\
(E+p) u
\end{array}\right)_{x}+\left(\begin{array}{c}
\rho v \\
\rho u v \\
\rho v^{2}+p \\
(E+p) v
\end{array}\right)_{y}=0,
$$

where the equation of state is

$$
p=(\gamma-1)\left(E-\frac{1}{2} \rho\left(u^{2}+v^{2}\right)\right) .
$$

1. Double Mach reflection of a strong shock.

This problem describes a Mach 10 shock reflected from the wall with an incidence angle of $60^{\circ}$. The computational domain is chosen to be $[0,4] \times[0,1]$, and the initial conditions are

$$
(\rho, u, v, p)= \begin{cases}(8.0,7.145,-4.125,116.5), & x<\frac{1}{6}+\frac{y}{\sqrt{3}} \\ (1.4,0,0,1), & x \geq \frac{1}{6}+\frac{y}{\sqrt{3}} .\end{cases}
$$




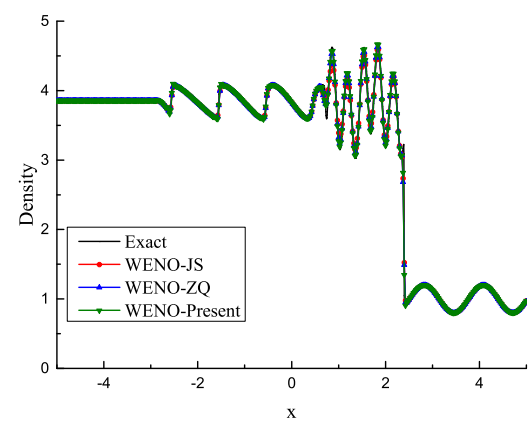

(a) Density

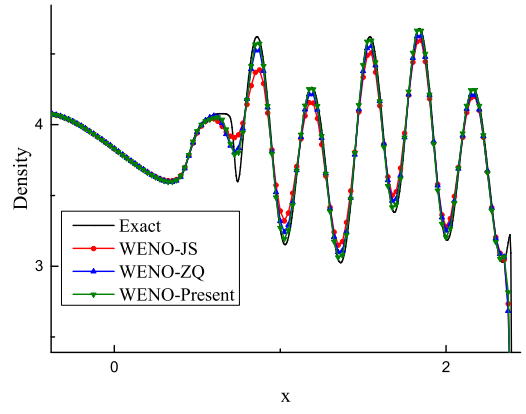

(b) Enlarged portion

Figure 8: Numerical solution of the Shu-Osher problem computed by the WENO-JS, WENO-ZQ and WENOPresent schemes. The figures show the density of the solution at $t=1.8$. $N=400$ points were used.

The ratio of specific heats is $\gamma=1.4$. We run the computation until $t=0.2$. Fig. 9 shows the results of WENO-Present and WENO-JS on the computation region with $960 \times 240$ grid points. Details of the region around the double Mach stems are shown in Fig. 10. It can be seen that the WENO-Present scheme resolves the instabilities better around the Mach stem than the WENO-JS scheme.

2. Rayleigh-Taylor instability.

This problem describes the flow motions on the interface between fluids of different densities. The heavy fluid moves into the region of the light fluid with a fingering nature, causing the bubbles of the light fluid to rise into the heavy fluid and the spikes of the heavy fluid to fall into the light fluid. The computational domain is $\left[0, \frac{1}{4}\right] \times[0,1]$, and the initial condition is

$$
(\rho, u, v, p)= \begin{cases}\left(2,0,-0.025 \sqrt{\frac{\gamma p}{\rho}} \cos (8 \pi x), 2 y+1\right), & 0 \leq y<\frac{1}{2} \\ \left(1,0,-0.025 \sqrt{\frac{\gamma p}{\rho}} \cos (8 \pi x), y+1.5\right), & \frac{1}{2} \leq y \leq 1\end{cases}
$$

where the ratio of specific heats is $\gamma=\frac{5}{3}$, and reflection boundary conditions are imposed for the left and right boundaries. The top boundary is set as $(\rho, u, v, p)=(1,0,0,2.5)$, and $(\rho, u, v, p)=(2,0,0,1)$ is the bottom boundary condition. The final time is $t=1.95$.

The solutions are computed by the WENO-JS, WENO-ZQ and WENO-Present schemes with meshes of $100 \times 400$, as shown in Fig. 11. Like the double Mach reflection of the strong shock problem, the WENO-Present scheme also possesses a higher resolution for capturing the solution than the other schemes. 


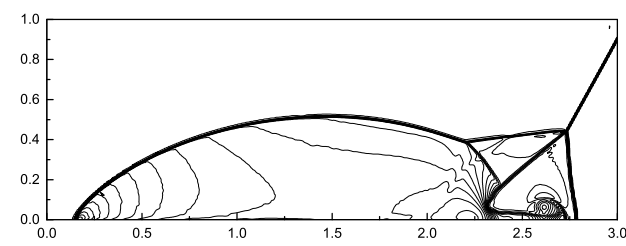

(a) WENO-JS

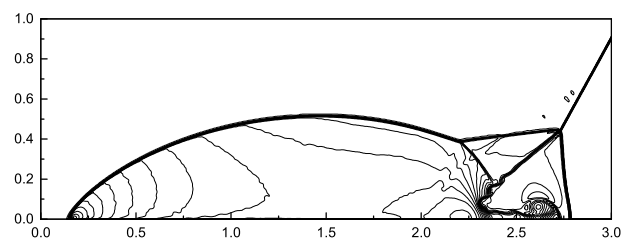

(b) WENO-ZQ

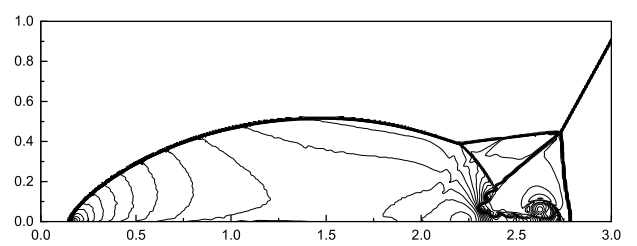

(c) WENO-Present

Figure 9: The density of Double Mach reflection computed by the WENO-JS, WENO-ZQ and WENO-Present schemes, and with 40 equally spaced contour lines from 1.8 to 20 .

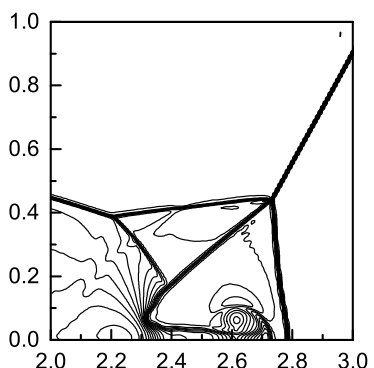

(a) WENO-JS

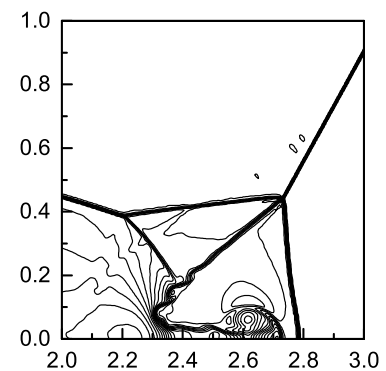

(b) WENO-ZQ

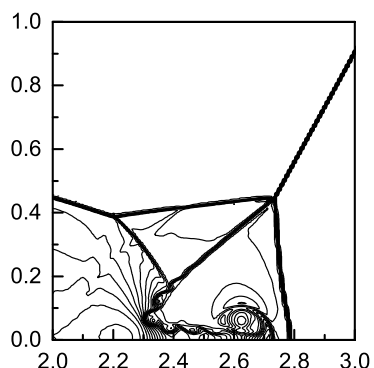

(c) WENO-Present

Figure 10: The local enlarged density distributions around the triple point computed by the WENO-JS, WENOZQ and WENO-Present schemes, with 40 equally spaced contour lines from 1.8 to 20.

\subsection{One-dimensional ideal dam-break simulation}

For the one-dimensional ideal dam-break model (Fig. 12), the computational region is $[0,1]$, and the vertical height of the bottom topography is $b=0$. Accordingly, the equations 


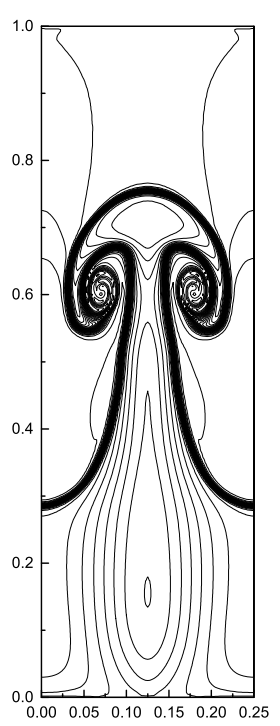

WENO-JS

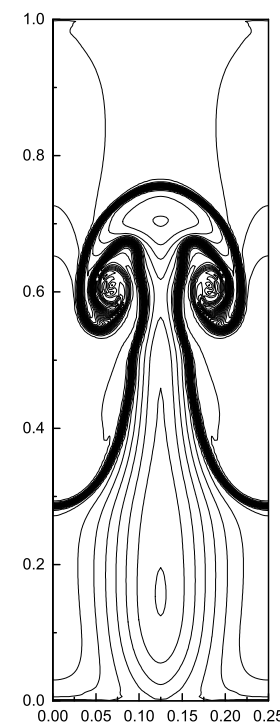

WENO-ZQ

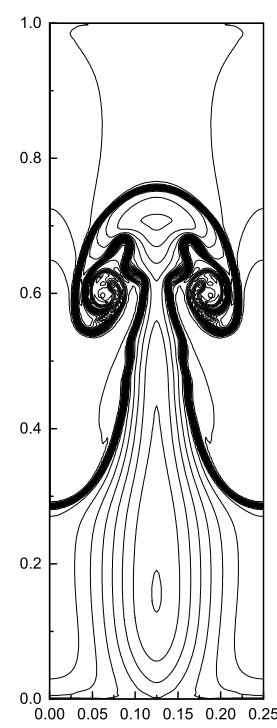

WENO-Present

Figure 11: The density contour of the Rayleigh-Taylor instability computed by the WENO-JS, WENO-ZQ and WENO-Present schemes with 30 contours in the range from 0.865 to 2.235 .

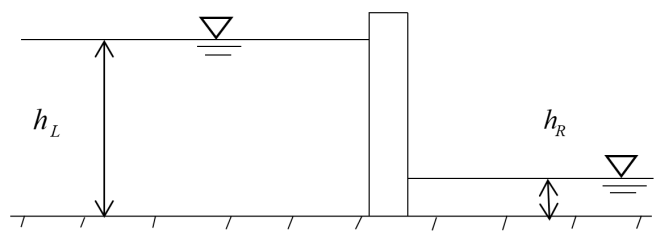

Figure 12: One-dimensional ideal dam-break test model diagram.

are obtained from $(1.1 b)$ :

$$
\begin{aligned}
& \frac{\partial h}{\partial t}+\frac{\partial(h u)}{\partial x}=0, \\
& \frac{\partial(h u)}{\partial t}+\frac{\partial\left(h u^{2}+\frac{1}{2} g h^{2}\right)}{\partial x}=0,
\end{aligned}
$$

with the initial condition

$$
\left\{\begin{array}{lll}
h_{L}=1.0, & u_{L}=0.0, & x \leq 0.6 \\
h_{R}=0.2, & u_{R}=0.0, & x>0.6
\end{array}\right.
$$

Fig. 13 provides the depth distribution at 0.08 after a dam break with $N=200$. Fig. 14 shows the velocity distribution at 0.08 after a dam break with $N=200$. According to the behavior of the computational parameters near the shock for Figs. 13 and 14, we can see that all the schemes simulate the dam-break flow very well, however, the WENO-Present scheme has the advantage of high-order accuracy. 


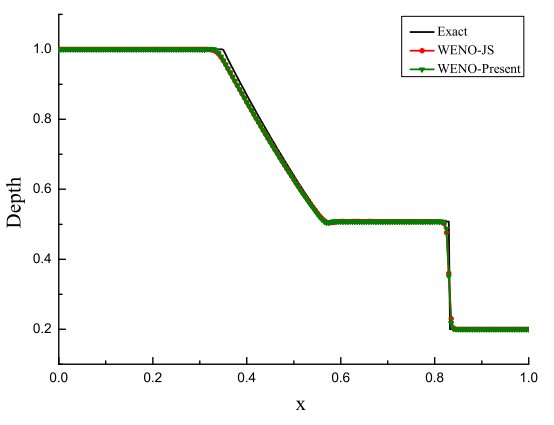

(a) Depth

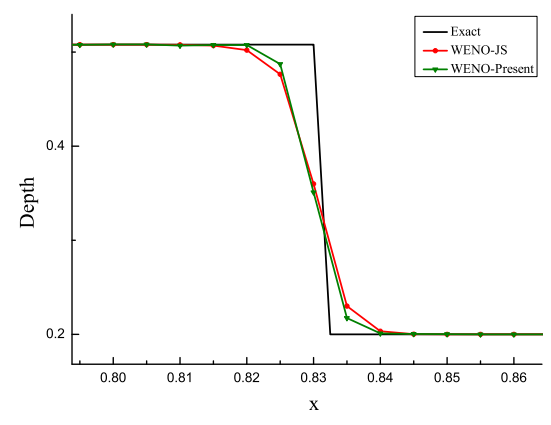

(b) Enlarged portion

Figure 13: Water surface profile distribution at $t=0.08 \mathrm{~s}$ after dam-break with $N=200$.

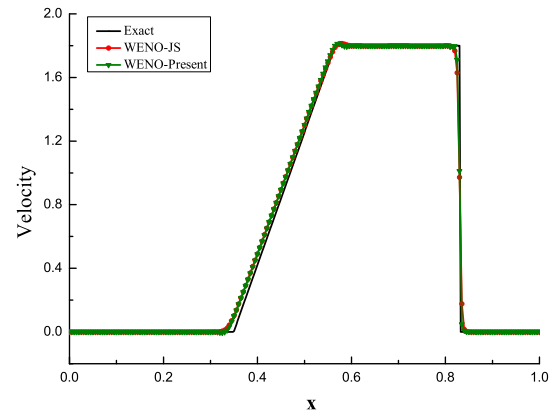

(a) velocity

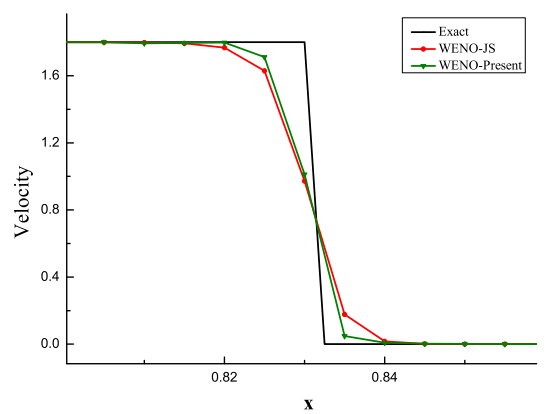

(b) Enlarged portion

Figure 14: Water velocity distribution at $t=0.08 \mathrm{~s}$ after dam-break with $N=200$.

\subsection{Two-dimensional ideal dam-break simulation}

For the two-dimensional ideal dam-break model, the failure of a dam in a $200 \times 200 \mathrm{~m}$ basin of simplified geometry is depicted in Fig. 15. The dam site is $100 \mathrm{~m}$ upstream, with a dam width of $10 \mathrm{~m}$. The problem models a partial dam break or the rapid opening of a sluice gate. At the instant of dam failure, water is released through a breach $75 \mathrm{~m}$ wide, forming a bore wave that propagates forward while spreading laterally. At the same time, a negative depression wave spreads upstream. The vertical height of the bottom topography is $b=0$. Accordingly, the following equations are obtained:

$$
\begin{aligned}
& \frac{\partial h}{\partial t}+\frac{\partial(h u)}{\partial x}+\frac{\partial(h v)}{\partial y}=0 \\
& \frac{\partial(h u)}{\partial t}+\frac{\partial\left(h u^{2}+\frac{1}{2} g h^{2}\right)}{\partial x}+\frac{\partial(h u v)}{\partial y}=0
\end{aligned}
$$




$$
\frac{\partial(h v)}{\partial t}+\frac{\partial(h u v)}{\partial x}+\frac{\partial\left(h v^{2}+\frac{1}{2} g h^{2}\right)}{\partial y}=0
$$

with the initial condition

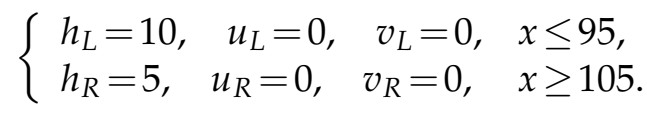

A $40 \times 40$ cell rectangular grid was chosen in this case, taking into account the regular shape of the domain of integration. Fig. 16 shows a 3D view of the water surface elevation, as obtained at time $t=7.2 \mathrm{~s}$, when the waves have not yet reached all the boundaries. Fig. 17 provides a map of level lines for the depth. These lines manifest as abrupt depressions in the water surface elevation in these regions. As can be observed, the numerical solutions of the WENO-JS and WENO-Present schemes exhibit stability and good behavior everywhere, but the WENO-Present scheme has a better simulation effect.

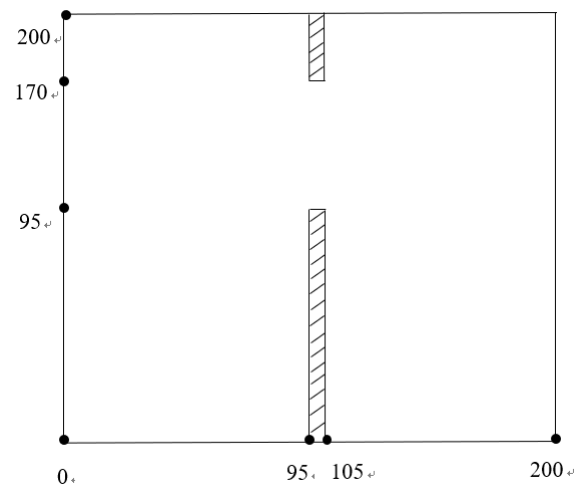

Figure 15: Two-dimensional ideal dam-break test model diagram.

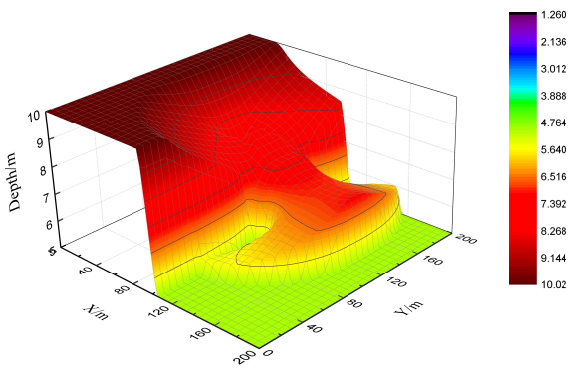

(a) WENO-JS

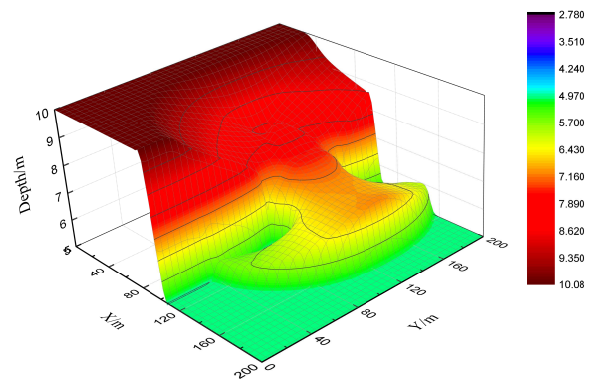

(b) WENO-Present

Figure 16: Water surface elevation at $t=7.2 \mathrm{~s}$ after dam-break. 


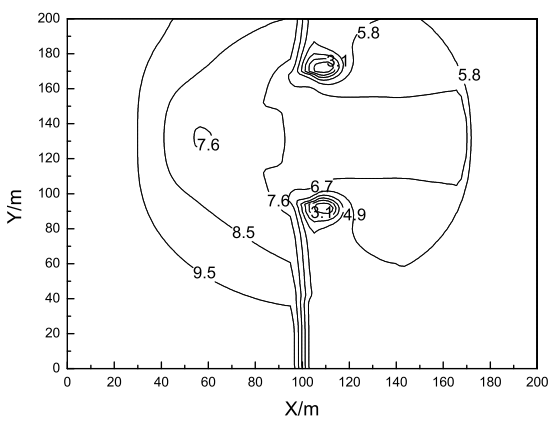

(a) WENO-JS

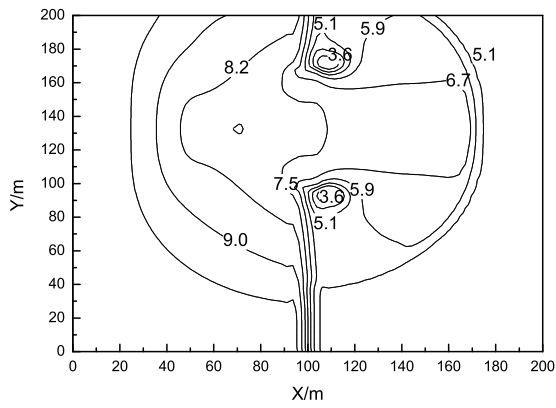

(b) WENO-Present

Figure 17: Contour plot showing depth distribution for partial dam-break test case.

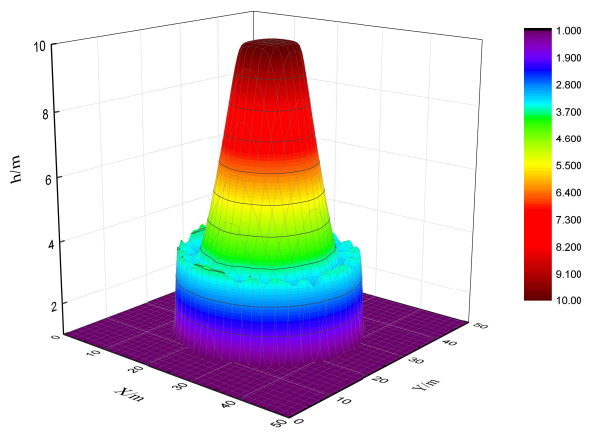

(a) WENO-JS

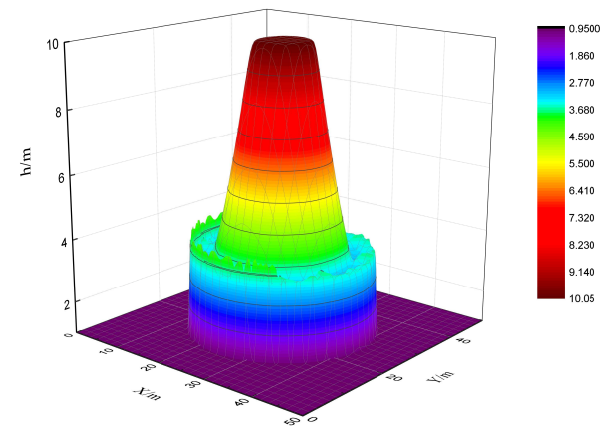

(b) WENO-Present

Figure 18: Water surface elevation at $t=0.55 \mathrm{~s}$ after dam-break.

Another interesting test case for assessing algorithm performance is the breaking of a dam with cylindrical symmetry and the subsequent time evolution of the wave pattern. The dam in a $50 \times 50 \mathrm{~m}$ region has the initial conditions

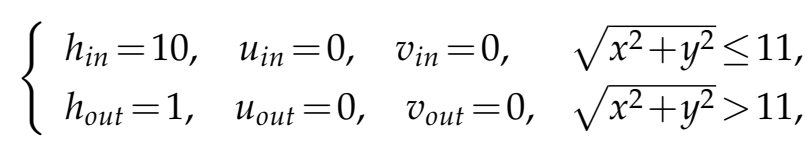

where two regions of still water are separated by a cylindrical wall (radius $=11 \mathrm{~m}$ ) with one region on the inner side of $h_{\text {in }}=10 \mathrm{~m}$ and the other region $h_{\text {out }}=1 \mathrm{~m}$ outside the dam. All the boundaries were assumed to be rigid.

The numerical results obtained with the new fifth-order scheme (WENO-Present) and the WENO-JS scheme on each of the grids for time $t=0.55 \mathrm{~s}$ are shown in Fig. 17. This figure shows a perspective view plot of the free-surface results computed in the rectangular grid. The irregularities around the propagating front and in the zone between it and 


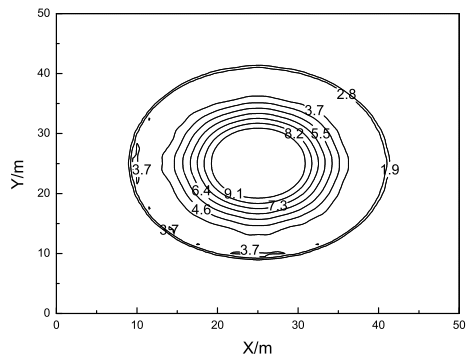

(a) WENO-JS

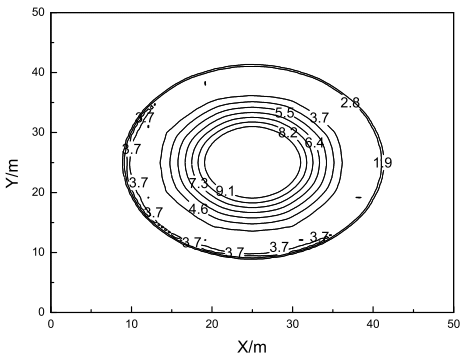

(b) WENO-Present

Figure 19: Contour plot showing depth distribution for the circular dam-break test case.

the depression wave mentioned above are visible in this image. Fig. 19 represents the respective level line maps of the depth. The results produced in the rectangular mesh are somewhat square, although perfect circular symmetry is expected. These observations illustrate that the WENO-Present scheme adequately simulates the breaking of a circular dam.

\subsection{The dam-breaking problem with nonflat bottom topography}

In this section, we combine the WENO-Present scheme with the well-balanced method, which is presented in [2] to simulate the dam-breaking problem with nonflat bottom topography. The detailed information about the well-balanced method for the shallow water equation can be seen in $[2,6]$.

\subsubsection{Test for the exact C-property}

First, we should verify that the well-balanced WENO-Present scheme maintains the exact C-property over a nonflat bottom, two functions for the bottom topography are given by $(0 \leq x \leq 10)$ :

$$
b(x)=5 e^{-\frac{2}{5}(x-5)^{2}},
$$

which is smooth, and

$$
b(x)= \begin{cases}4, & \text { if } 4 \leq x \leq 8, \\ 0, & \text { otherwise }\end{cases}
$$

which is discontinuous. The initial condition is the stationary solution:

$$
h+b=10, \quad h u=0 .
$$

We compute the solution using the well-balanced WENO-Present scheme with $N=$ 100,200 and 400 cells, the final time is $t=0.5$. The computed surface level $h+b$ and bottom $b$ are plotted in Fig. 20. 


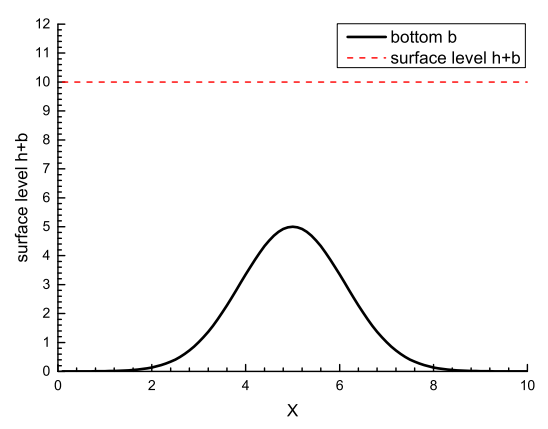

(a) Smooth bottom

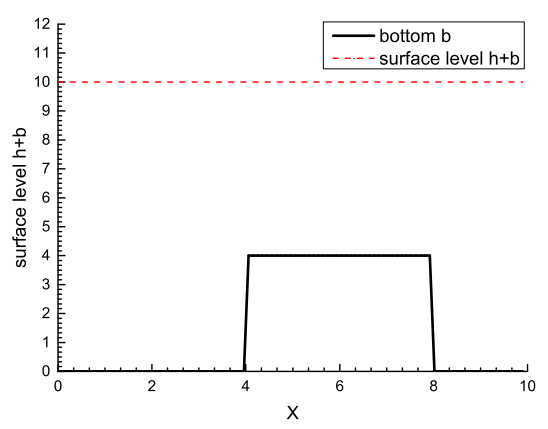

(b) Discontinuous bottom

Figure 20: The surface level $h+b$ and the bottom $b$ for stationary flow.

The double precision is used to perform the computation, $L_{\infty}$ and $L_{2}$ errors for the surface level $h+b$ and water discharge $h u$ are shown in Tables 7 and 8 . It can be seen that the $L_{\infty}$ and $L_{2}$ errors for both the smooth and discontinuous bottom topographies are at level of round-off errors, therefore, the WENO-Present scheme can preserve the exact C-property.

Table 7: $L_{\infty}$ and $L_{2}$ errors in the water surface level $h+b$ and water discharge $h u$ as computed by the WENOPresent scheme with the smooth bottom topography (3.19).

\begin{tabular}{||c|cccc||}
\hline \multirow{2}{*}{$N$} & \multicolumn{2}{|c}{$L_{\infty}$ error } & \multicolumn{2}{c||}{$L_{2}$ error } \\
\cline { 2 - 5 } & $h+b$ & $h u$ & $h+b$ & $h u$ \\
\hline 100 & $9.38 \mathrm{e}-14$ & $8.56 \mathrm{e}-13$ & $5.24 \mathrm{e}-14$ & $5.86 \mathrm{e}-13$ \\
200 & $2.07 \mathrm{e}-13$ & $1.79 \mathrm{e}-12$ & $1.10 \mathrm{e}-13$ & $1.24 \mathrm{e}-12$ \\
400 & $4.15 \mathrm{e}-13$ & $3.76 \mathrm{e}-12$ & $2.15 \mathrm{e}-13$ & $2.49 \mathrm{e}-12$ \\
\hline
\end{tabular}

Table 8: $L_{\infty}$ and $L_{2}$ errors in the water surface level $h+b$ and water discharge $h u$ as computed by the WENOPresent scheme with the smooth bottom topography (3.20).

\begin{tabular}{||c|cccc||}
\hline \multirow{2}{*}{$N$} & \multicolumn{2}{|c}{$L_{\infty}$ error } & \multicolumn{2}{c||}{$L_{2}$ error } \\
\cline { 2 - 5 } & $h+b$ & $h u$ & $h+b$ & $h u$ \\
\hline 100 & $4.66 \mathrm{e}-14$ & $1.93 \mathrm{e}-13$ & $2.66 \mathrm{e}-14$ & $1.27 \mathrm{e}-13$ \\
200 & $9.15 \mathrm{e}-13$ & $3.49 \mathrm{e}-12$ & $4.09 \mathrm{e}-14$ & $3.83 \mathrm{e}-13$ \\
400 & $2.85 \mathrm{e}-13$ & $9.36 \mathrm{e}-12$ & $1.11 \mathrm{e}-14$ & $7.05 \mathrm{e}-13$ \\
\hline
\end{tabular}

\subsubsection{The dam-breaking problem over a rectangular bump}

In this example, the dam-breaking problem over a rectangular bump is chosen to investigate the capability of the WENO-Present scheme in shock capturing, this classical example was also used in $[1,2,6]$. 


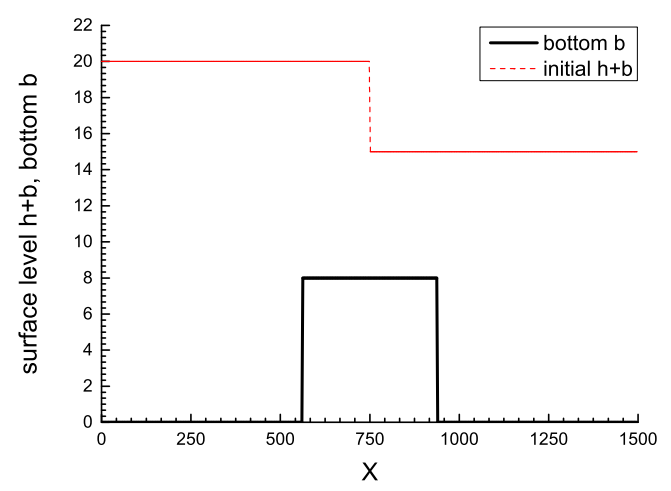

Figure 21: The initial surface level $h+b$ and bottom topography $b$ for the dam-breaking problem.

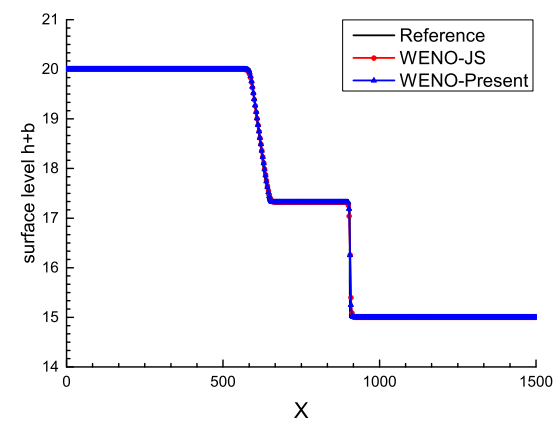

(a) Surface level $h+b$

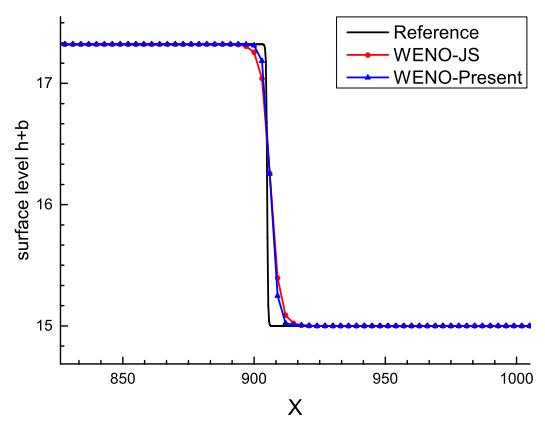

(b) Enlarged portion

Figure 22: The surface level $h+b$ for the dam-breaking problem at time $t=15 \mathrm{~s}$.

The bottom topography is given by

$$
b(x)= \begin{cases}8, & \text { if }|x-750| \leq 1500 / 8 \\ 0, & \text { otherwise }\end{cases}
$$

for $x \in[0,1500]$. The initial conditions are:

$$
(h u)(x, 0)=0 \quad \text { and } \quad h(x, 0)= \begin{cases}20-b(x), & \text { if } x \leq 750 \\ 15-b(x), & \text { otherwise }\end{cases}
$$

The initial surface level $h+b$ and the bottom $b$ are plotted in Fig. 21. The final surface levels $h+b$ computed by the WENO-Present scheme with grid points $N=500$ at times $t=15 \mathrm{~s}$ and $t=60 \mathrm{~s}$ are plotted in Figs. 22 and 23, the reference solution is computed by the WENO-JS scheme with grid points $N=5000$. The good performance of the WENOPresent scheme is demonstrated in the figures, and the final surface levels $h+b$ at different times are in good agreement with the results of $[2,6]$. 


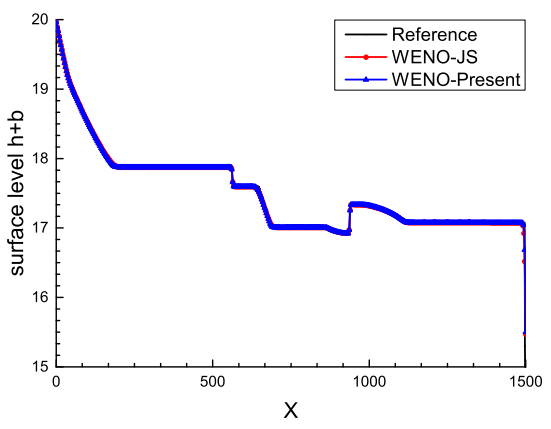

(a) Surface level $h+b$

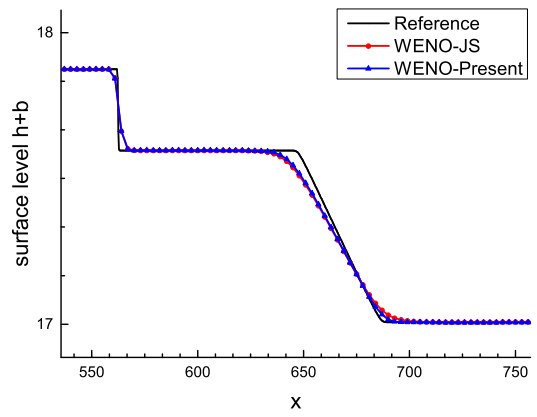

(b) Enlarged portion

Figure 23: The surface level $h+b$ for the dam-breaking problem at time $t=60$ s.

\section{Conclusions}

In this paper, we derived a simple fifth-order finite difference WENO scheme called WENO-Present for dam-break simulations. The WENO-Present scheme uses the information that lies in one five-point stencil and two smaller three-point stencils, thus, the scheme makes use of more information contained in the point than does the WENO-ZQ scheme, smaller error and higher precision were obtained by the WENO-Present scheme than by the WENO-ZQ scheme. Another advantage of the WENO-Present scheme is its simplicity. The construction of WENO-Present is based on WENO interpolation in spatial fields, after which a third-order TVD Runge-Kutta time discretization procedure is used to solve the ODE. Compared with classical WENO schemes, the WENO-Present scheme is very simple in the computation of problems with shocks. Meanwhile, the proposed scheme can obtain the same accuracy order. Our analysis also indicates that the WENO-Present scheme has an excellent resolution over the WENO-JS scheme when solving problems with large gradients and shocks. Finally, one- and two-dimensional ideal dam-break flow, dam breaking with a nonflat bottom topography are tested by the WENO-Present scheme, and the efficiency and stability of this scheme are verified.

\section{Acknowledgements}

This research was supported by National Natural Science Foundation of China (grant No. 51579206), the Key R \& D Projects in Shaanxi Province, China (grant No. 2018SF352), the Water Conservancy Science and Technology Project of Shaanxi Province, China (grant No. 2017slkj-17). 


\section{References}

[1] S. VUKOVIC AND L. SOPTA, ENO and WENO schemes with the exact conservation property for one-dimensional shallow water equations, J. Comput. Phys., 179 (2002), pp. 593-621.

[2] Y. L. XING AND C. W. SHU, High order finite difference WENO scheme with the exact conservation property for the shallow water equations, J. Comput. Phys., 208 (2005), pp. 206-227.

[3] J. G. Zhou, D. M. CAuson, C. G. Mingham and D. M. Ingrma, The surface gradient method for the treatment of source terms in the shallow-water equations, J. Comput. Phys., 168 (2001), pp. 1-25.

[4] G. Thierry, J. M. Herard AND N. Seguin, Some approximate Godunov schemes to compute shallow-water equations with topography, Comput. Fluids, 32 (2003), pp. 479-513.

[5] G. LI, V. CALEFFI AND J. M. GAO, High-order well-balanced central WENO scheme for prebalanced shallow water equations, Comput. Fluids, 99 (2014), pp. 182-189.

[6] Z. GAO AND G. H. HU, High order well-balanced weighted compact nonlinear schemes for shallow water equations, Commun. Comput. Phys., 22 (2017), pp. 1049-1068.

[7] Q. Q. ZHU, Z. GAO, W. S. DON AND X. Q. LV, Well-balanced hybrid compact-WENO scheme for shallow water equations, Appl. Numer. Math., 112 (2017), pp. 65-78.

[8] X. F. WANG, G. LI, S. G. QIAN, J. J. Li AND Z. WANG, High order well-balanced finite difference WENO schemes for shallow water flows along channels with irregular geometry, Appl. Math. Comput., 363 (2019), pp. 1-16.

[9] Z. M. WANG, J. ZHU AND N. ZHAO, A new robust high-order weighted essentially nonoscillatory scheme for solving well-balanced shallow water equations, Adv. Appl. Math. Mech.,11 (2019), pp. 911-941.

[10] A. HARTEN, High resolution schemes for hyperbolic conservation laws, J. Comput. Phys., 49 (1983), pp. 357-393.

[11] A. HARTEN AND S. OSHER, Uniformly high order accurate essentially non-oscillatory schemes I, SIAM J. Numer. Anal., 24 (1987), pp. 279-309.

[12] A. HARTEN, B. ENGQUiST AND S. OsHER, Uniformly high order accurate essentially nonoscillatory schemes III, J. Comput. Phys., 131 (1987), pp. 3-47.

[13] Y. T. ZHANG AND C. W. SHU, Third order WENO scheme on three dimensional tetrahedral meshes, Commun. Comput. Phys., 5 (2008), pp. 836-848.

[14] A. HaRTEN, High resolution schemes for hyperbolic conservation laws, J. Comput. Phys., 135 (1997), pp. 260-278.

[15] R. Borges, M. Carmona, B. Costa And W. S. Don, An improved weighted essentially nonoscillatory scheme for hyperbolic conservation laws, J. Comput. Phys., 227 (2008), pp. 3191-3211.

[16] F. X. ZHAO, L. PAN, Z. Li AND S. H. WANG, A new class of high-order weighted essentially non-oscillatory schemes for hyperbolic conservation laws, Comput. Fluids, 159 (2005), pp. 81-94.

[17] D. SHAW, S. GARAIN AND C. W. SHU, An efficient class of WENO schemes with adaptive order, J. Comput. Phys., 326 (2016), pp. 780-804.

[18] A. HARTEN, On a class of high resolution total-variation-stable finite-difference schemes, SIAM J. Numer. Anal., 21 (1984), pp. 1-23.

[19] S. OSHER AND S. CHAKRAVARTHY, High resolution schemes and the entropy condition, SIAM J. Numer. Anal., 21 (1984), pp. 955-984.

[20] X. D. LiU, S. Osher AND T. CHAN, Weighted essentially non-oscillatory schemes, J. Comput. Phys., 115 (1994), pp. 200-212.

[21] G. S. JIANG AND C. W. SHU, Efficient implementation of weighted ENO schemes, J. Comput. Phys., 126 (1996), pp. 202-228. 
[22] G. S. JiAnG AND C. W. SHU, Efficient implementation of essentially non-oscillatory shockcapturing schemes, J. Comput. Phys., 77 (1998), pp. 439-471.

[23] S. ZHANG, S. JiANG AND C. W. SHU, Development of nonlinear weighted compact schemes with increasingly higher order accuracy, J. Comput. Phys., 227 (2008), pp. 7294-7321.

[24] J. X. QIU AND C. W. SHU, Hermite WENO schemes for hamilton-jacobi equations, J. Comput. Phys., 204 (2005), pp. 82-99.

[25] S. RATHAN AND G. NAGA RAIU, A modified fifth-order scheme for hyperbolic conservation laws, Comput. Math. Appl., 5 (2018), pp. 1531-1549.

[26] W. Z. XU AND W. G. WU, Improvement of third-order WENO-Z scheme at critical points, Comput. Math. Appl., 9 (2018), pp. 3431-3452.

[27] C. W. SHU, High-order finite difference and finite volume WENO schemes and discontinuous galerkin methods for CFD, Int. J. Comput. Fluid Dyn., 17 (2003), pp. 107-118.

[28] Q. ZHOU, F. HE AND M. Y. SHEN, A family of efficient high-order hybrid finite difference schemes based on WENO schemes, Int. J. Comput. Fluid Dyn., 26 (2012), pp. 205-229.

[29] R. Abedian, H. Adibi AND M. DEHGHAN, Symmetrical weighted essentially non-oscillatoryflux limiter schemes for Hamilton-Jacobi equations, Math. Methods Appl. Sci., 38 (2016), pp. $4710-4728$.

[30] W. S. DON, P LI, K. Y. WONG AND Z. GAO, Improved symmetry property of high order weighted essentially non-oscillatory finite difference schemes for hyperbolic conservation laws, Adv. Appl. Math. Mech., 10(6) (2018), pp. 1418-1439.

[31] A. K. Henrick, T. D. Aslam And J. M. POWERs, Mapped weighted essentially non-oscillatory schemes: Achieving optimal order near critical points, J. Comput. Phys., 207 (2005), pp. 542-567.

[32] M. CASTRO, B. COSTA AND W. S. DON, High order weighted essentially non-oscillatory WENOZ schemes for hyperbolic conservation laws, J. Comput. Phys., 230 (2011), pp. 1766-1792.

[33] J. ZHU AND J. X. QIU, A new fifth order finite difference WENO scheme for solving hyperbolic conservation laws, J. Comput. Phys., 318 (2016), pp. 110-121. 